\title{
Costs and benefits of strategy construction in a speeded discrimination task
}

\author{
GORDON D. LOGAN \\ Erindale College, Mississauga, Ontario, Canada \\ N. JANE ZBRODOFF \\ Ontario Institute for Studies in Education, Toronto, Ontario, Canada \\ and \\ ALAN R. W. FOSTEY \\ Erindale College, Mississauga, Ontario, Canada
}

\begin{abstract}
The costs and benefits of constructing and utilizing a strategy for performing a speeded discrimination task were assessed in terms of time and mental resources. The strategy involved using information about the position a word occupied in order to respond to its identity more rapidly. The resources used to construct and utilize the strategy were assessed from responses to probes presented at various times during the task. In Experiment 1, the probe required a manual response, whereas in Experiment 2, the probe required a vocal response. Strategy construction interfered with the probe task in Experiment 1 but not in Experiment 2, suggesting that strategy construction required resources specific to the manual response system. There was also some evidence that strategy construction required general resources, but the evidence for specific resources was much stronger. The findings are consistent with our previous ideas about how the strategy is represented (Logan, 1980a; Logan \& Zbrodoff, 1979). The different results with different responses to the probes point out important limitations on previous studies of probe interference and of cost-benefit analysis, which each focused on a single dimension of cost and benefit. The differences suggest that a multiple-resources approach to probe interference and cost-benefit analysis might be more appropriate.
\end{abstract}

In general terms, a strategy may be defined as an optional organization of cognitive processes that is intended to achieve some goal in some task environment. The strategy chosen for a particular task is probably a compromise between the constraints imposed by the structure of the information in the task environment, by the structure of the subject's cognitive abilities, and by the structure of the goals to be achieved by performing the task. Probably, several strategies can satisfy the constraints for any given task, but only one can be used each trial. How is the one chosen? The different possibilities can be ranked in terms of the benefits the subject can expect to gain from them and the costs associated with attaining the benefits. Then, the strategy that maximizes benefit and minimizes cost is the best choice.

This research was supported by Grant U0053 from the Natural Sciences and Engineering Research Council of Canada to Gordon Logan. Jane Zbrodoff was supported by a doctoral fellowship from the Social Sciences and Humanities Research Council of Canada. We are grateful to Ken Davis for running the subjects and to Steven Keele, Peter McLeod, and an anonymous reviewer for helpful comments on the manuscript. Requests for reprints may be addressed to Gordon D. Logan, who is now with the Department of Psychology, University of British Columbia, Vancouver, British Columbia V6T 1W5, Canada.
Current cost-benefit analyses of strategies in information-processing tasks focus on temporal aspects of strategy utilization, balancing the reduction in reaction time that results from using the strategy appropriately against the increase in reaction time that results from using the strategy inappropriately (e.g., Posner \& Snyder, 1975). This perspective may be limited in two important respects: First, it considers only one dimension of cost and benefit: the time taken to use the strategy. Possibly, cost and benefit can vary in dimensions other than time. Each mental resource required to construct or utilize a strategy may constitute a separate dimension along which cost and benefit may vary, and the demand for various resources may affect the choice of strategies (see Navon \& Gopher, 1979, and Wickens, 1980). Second, current cost-benefit analyses focus on the costs and benefits of utilizing the strategy once an appropriate stimulus appears but say little about the costs and benefits of constructing the strategy in preparation for an upcoming stimulus (but see McLean \& Shulman, 1978). The mental operations involved in organizing processes in a way that will achieve the goals of performing probably take time and resources, which may be balanced against the time and resources saved by using the strategy when the overall cost and benefit are assessed. 
The purpose of the present article is to evaluate the costs and benefits of strategy construction and utilization in terms of mental resources, as well as in terms of time. The findings demonstrate important limits on previous cost-benefit analyses, which have focused primarily on time gained and lost in strategy utilization. The findings also provide an assessment of the costs and benefits associated with particular strategies for performing speeded discrimination tasks that we have been investigating for the past few years (Logan, 1980a; Logan \& Zbrodoff, 1979, 1982). Following Posner's (1978) lead in using simple "model" systems to study complex attentional phenomena, we expect that the principles governing strategy construction and utilization in our speeded discrimination task will generalize to richer task environments.

\section{Strategic Attention to Unreported Dimensions}

The strategies under investigation involved combining information from different dimensions of a multidimensional stimulus. The task was to report the identity of a word as quickly and as accurately as possible. On each trial, the word ABOVE or the word BELOW appeared above or below a fixation point at the center of a viewing screen. In order to respond appropriately, the subjects had to attend selectively to the identity of the word and to ignore its position on the screen. On some trials, the subjects were informed of the relation between position and identity (i.e., whether the dimensions and compatible meanings, e.g., ABOVE/above or $\mathrm{BELOW} / \mathrm{below}$, or conflicting meanings, e.g., ABOVE/ below or BELOW/above), and two more options became available for performing the task appropriately: The subjects could divide attention between dimensions, basing their responses partly on the identity of the word and partly on its position, or they could attend selectively to the position of the word and ignore its identity. With the stimuli used in those experiments, the subjects could respond to the word's position faster than they could respond to the word's identity (see Logan \& Zbrodoff, 1979, Experiment 1), so they could gain speed by attending only to position or by dividing attention between dimensions.

There are two ways to inform subjects about the relation between dimensions: First, the relative frequency of compatible and conflicting trials can be varied to correlate the dimensions, and subjects can be told about the correlation in advance or they can be given enough trials to induce it themselves (see Greenwald \& Rosenberg, 1978, Logan, 1980a, and Logan \& Zbrodoff, 1979). Second, a precue can be given on each trial to indicate whether the dimensions of the upcoming word will be compatible or conflicting (see Logan \& Zbrodoff, 1982). With this procedure, subjects must construct the strategy anew each trial, so the construction process can be observed more easily.

Previous studies with a precue suggest there are substantial time costs involved in strategy construction that outweigh the temporal benefits. Logan and Zbrodoff (1982) measured the time required to construct strategies by varying the delay between the onset of the cue and the onset of the word. They found that benefit increased as cue delay increased from 100 to $1,000 \mathrm{msec}$, but reached an asymptote of $50-100 \mathrm{msec}$ at delays of $400-600 \mathrm{msec}$. This suggests that $400-600 \mathrm{msec}$ were required to construct the strategy, which is a substantial cost compared with the 50-100-msec benefit that resulted from using the strategy. However, the time required for construction may not represent cost. Automatic processes take time, yet are cost free (Posner \& Boies, 1971 ; Posner \& Snyder, 1975) or, at least, relatively cheap (Navon \& Gopher, 1979). The time spent in strategy construction can be considered costly only to the extent that it prevents subjects from pursuing other activities that may be beneficial. This fact is what motivated the present dual-task study of the mental resources involved in strategy construction and utilization.

\section{Mental Resources and the Probe Task}

The experiments reported here used a probe task to investigate the mental resources used in strategy construction and utilization. On each trial, the subjects were given a precue followed by the word ABOVE or BELOW, which they were to identify. The subjects were also asked to respond to probes presented during the presentation of the precue or the word, and their reaction times to the probes were used as a measure of the resources required for strategy construction and utilization (see Posner \& Boies, 1971). The resources involved in constructing the strategy were assessed from reaction times to probes presented with the cue. If construction requires resources, probe reaction times should be longer when the cue is valid (hence, subjects construct strategies) than when the cue is neutral (hence, subjects do not construct strategies). Similarly, the resources involved in strategy utilization were assessed from differences between reaction times to probes presented with the word in the cue-valid and cue-neutral conditions.

It is often assumed that differences in probe reaction times reflect the use of a general attentional resource, and the probe task has gained wide currency as a method for measuring the attention demands of mental activities (e.g., Comstock, 1973; McLean \& Shulman, 1978; Paap \& Ogden, 1981; Posner \& Boies, 1971; Posner \& Klein, 1973; Proctor \& Fisicaro, 1977). However, recent theory and data suggest that the probe task may reflect other resources in addition to general attentional capacity.

Multiple-resource theory argues that performance is limited by several different resources, in addition to general attentional capacity (e.g., Allport, 1980; Navon \& Gopher, 1979; Wickens, 1980). Concurrent tasks may compete for several resources, so performance deficits in dual-task situations cannot be traced back to a particular resource without ambiguity. This challenges 
interpretations of probe interference in terms of demand for attention. However, it is possible to identify the resources used in a particular task by having that task performed concurrently with several tasks that differ in the resources they require. Some investigators have used different versions of the probe task to assess specific resource demands, and different patterns of interference have emerged, depending on the nature of the probe and the nature of the response to the probe. In visual matching tasks, for example, visual probes produce more interference than do auditory probes, suggesting that the matching task requires specific visual resources (Millar, 1975; Proctor \& Proctor, 1979; Shwartz, 1976). McLeod (1978) found that probes that required a vocal response produced a pattern of interference different from that produced by probes that required a manual response, suggesting that the matching task requires specific motor resources.

The present experiments used different combinations of probes and probe responses to identify the specific and general resources required for strategy construction and utilization. In both experiments, the probe was a tone, which was not expected to compete with the word task for specific perceptual resources. It was, however, expected to compete for general attentional resources. In Experiment 1, the probe required a manual response, which was expected to compete with the (manual) response to the word for specific motor resources as well as for general attentional resources. In Experiment 2 , the probe required a vocal response, which was not expected to compete with the response to the word for specific motor resources, although it was expected to compete for general attentional resources. With this design, patterns of interference that differ between experiments reflect the involvement of specific motor resources (see McLeod, 1978), whereas patterns of interference that are the same in the two experiments reflect the involvement of general resources, possibly attentional (see Posner \& Boies, 1971).

\section{EXPERIMENT 1}

\footnotetext{
Method

Subjects. Eight graduate and undergraduate students and laboratory staff from Erindale College served in the experiment for monetary compensation. Each subject served in four 1-h sessions.

Apparatus and Stimuli. The stimuli were the words ABOVE and BELOW, written in capitals and presented above and below a central fixation point, and the cues $X$ and $O$, also written in capitals but presented in the center of the screen. The stimuli were displayed on a point-plot cathode ray tube (Tektronix Model 604, equipped with P31 phosphor) under the control of a PDP11/03 laboratory computer. Each letter was formed by illuminating about 20 points in a $5 \times 7$ matrix and subtended about $.43 \times .57 \mathrm{deg}$ of visual angle when viewed at a distance of $60 \mathrm{~cm}$. Constant viewing distance was maintained by a headrest. Each word subtended about $2.68 \mathrm{deg}$ horizontally and $.57 \mathrm{deg}$ vertically, and appeared $2.77 \mathrm{deg}$ above or below the fixation point. The computer "painted" one point on the screen every 78 microsec. The brightness of the single-letter cues was
}

made the same as that of the five-letter words by painting four extra letters at a nonexistent position on the screen before refreshing the cue.

Each trial began with a central fixation point exposed for $500 \mathrm{msec}$. It was then extinguished and replaced by the cue, which was exposed for $500 \mathrm{msec}$. The cue was extinguished, and $100 \mathrm{msec}$ later, the word appeared, exposed for $500 \mathrm{msec}$. Following the word, the screen was blank for a $1,000-\mathrm{msec}$ intertrial interval.

The probe was a $900-\mathrm{Hz}, 500-\mathrm{msec}$ tone played through a speaker behind the cathode ray tube. It was presented on half of the trials, $400,550,700,850,1,000$, or $1,150 \mathrm{msec}$ after the beginning of the trial. The $400-\mathrm{msec}$ probe occurred before the cue appeared; the 550-, 700-, and $850-\mathrm{msec}$ probes occurred while the cue was exposed; the 1,000-msec probe began at the same time that the cue was extinguished; and the $1,150-\mathrm{msec}$ probe began $50 \mathrm{msec}$ after the word appeared.

The subjects responded to the word by pressing either the rightmost or the next-to-rightmost key of a row of eight telegraph keys mounted on a panel in front of them. Every subject pressed the next-to-rightmost key if the word's identity was "above" and the rightmost if the word's identity was "below." The subjects responded to the probe tone by pressing the leftmost key in the row of eight.

Procedure. Each session, each subject completed 768 trials, divided into four blocks of 192. For two of the blocks the cue was neutral, and for two of the blocks the cue was valid. Valid and neutral blocks alternated throughout the session to alleviate boredom. Within each block, the tone occurred on half of the trials (96) and was witheld on the other half. It occurred equally often (16 times) at each of the 6 delays $(400,550,700,850$, 1,000 , or $1,150 \mathrm{msec}$ after the beginning of the trial). Half of the time at each delay the tone occurred with compatible stimuli, and half of the time with conflicting stimuli. Half of the no-tone trials involved compatible stimuli, and half involved conflicting stimuli. When the cue was valid, $\mathrm{X}$ appeared before every compatible stimulus, and $O$ appeared before every conflicting stimulus. When the cue was neutral, $X$ appeared before compatible stimuli half of the time and before conflicting stimuli half of the time, and the same was true for $O$. In each condition, the two different compatible stimuli (i.e., ABOVE/ above and BELOW/below) occurred equally often and the two different conflicting stimuli (i.e., ABOVE/below and BELOW/ above) occurred equally often. The order of stimuli, probes, and probe delays was randomized within these constraints. A separate random order was prepared for each subject each session.

The order of cue-valid and cue-neutral blocks varied between and within subjects each session. On the first session, half of the subjects began with the cue valid and half began with the cue neutral. On subsequent sessions, each subject alternated between beginning with the cue valid and beginning with the cue neutral, continuing the cycle he or she had begun on the first session.

The subjects were instructed to report the identity of the word on each trial. When the cue was valid, they were told that $\mathrm{X}$ preceded compatible stimuli and $\mathrm{O}$ preceded conflicting stimuli, and that they should try to use the cue to improve their performance. They were told that we knew from previous research that subjects could respond faster if they used the cue, and they were encouraged to use it on every trial. Emphatic instructions such as these were necessary because, without them, subjects tend to ignore the cue despite its validity. When the cue was neutral, the subjects were told that $X$ and $O$ occurred before compatible stimuli as of ten as before conflicting stimuli, so they should ignore the cue.

After the instructions for the word task, the probe task was described. The subjects were told that a probe would occur occasionally and that they should respond to it as quickly as possible without slowing their performance on the word task. They were told explicitly to protect the word task from interference, to concentrate the bulk of the dual-task interference on the probe task. The difference in emphasis and frequency 
served to define the word task as primary and the probe task as secondary (see Posner \& Boies, 1971).

\section{Results}

Word task. Mean reaction times to the words from trials on which probe tones were presented and from trials on which the probes were withheld are plotted in Figure 1 as a function of cue validity, compatibility, and probe delay. Each of the points from probe trials is based on a maximum of 512 observations, and each of the points from no-probe trials is based on a maximum of 3,072 observations.

Overall, reaction times were $22 \mathrm{msec}$ faster when the cue was valid than when the cue was neutral $[F(1,7)=$ $29.40, \mathrm{p}<.01, \mathrm{MSe}=3,877.49]$, indicating that the subjects were able to construct and utilize a strategy that involved dividing attention between dimensions or attending exclusively to a word's position (see Logan \& Zbrodoff, 1982). Also, reaction times to conflicting stimuli were $15 \mathrm{msec}$ slower than reaction times to compatible stimuli $[\mathrm{F}(1,7)=10.43, \mathrm{p}<.05, \mathrm{MSe}=$ $4,920.24]$, reflecting a Stroop-type effect found previously with these stimuli (Logan \& Zbrodoff, 1979, 1982). The cue-validity effect tended to be stronger with compatible stimuli than with conflicting stimuli $(27 \mathrm{msec}$ vs. $18 \mathrm{msec}$ ), as had been found previously (Logan \& Zbrodoff, 1982), but the interaction between compatibility and cue validity was not significant $[F(1,7)=$ 2.13, $\mathrm{p}<.20, \mathrm{MSe}=2,063.78]$.

Overall, reaction time increased with probe delay $[F(6,42)=13.42, p<.01, \mathrm{MSe}=5,706.87]$, reflecting response interference between the processes responding to the probe and the processes responding to the word (i.e., strategy utilization). Reaction time to the word increased the closer together the responses were in time.

The cue-validity effect (i.e., the difference between cue-valid and cue-neutral reaction times) increased as probe delay increased $[\mathrm{F}(6,42)=5.21, \mathrm{p}<.01, \mathrm{MSe}=$ 835.53 ], reflecting interference with strategy construction. Apparently, responding to a probe presented at an

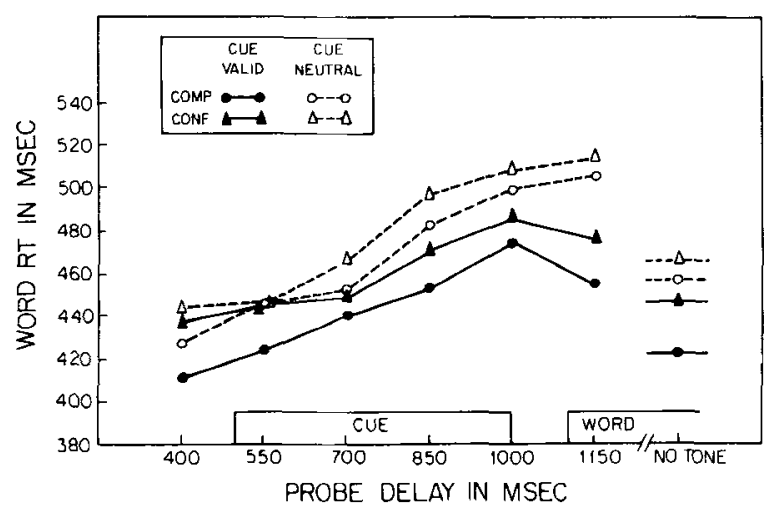

Figure 1. Reaction time to the word on no-tone trials and reaction time to the word on tone trials as a function of probe delay in Experiment 1 (compatibility and cue validity are the parameters comp = compatible conf $=$ conflicting).
Table 1

Proportion of Errors in the Word Task in Experiment 1 as a Function of Cue Validity, Compatibility, and Probe Delay

\begin{tabular}{llllllllll}
\hline \multirow{2}{*}{$\begin{array}{c}\text { Cue } \\
\text { Validity }\end{array}$} & $\begin{array}{l}\text { Compat- } \\
\text { ibility }\end{array}$ & \multicolumn{6}{c}{ Probe Delay (in Milliseconds) } & No \\
\cline { 3 - 7 } Valid & 400 & 550 & 700 & 850 & 1000 & 1150 & Tone \\
\hline \multirow{4}{*}{ Neutral } & Comp & .04 & .05 & .06 & .08 & .11 & .06 & .07 \\
& Conf & .05 & .07 & .04 & .07 & .10 & .09 & .07 \\
& Comp & .04 & .04 & .05 & .05 & .07 & .07 & .05 \\
& Conf & .04 & .03 & .03 & .04 & .08 & .07 & .05 \\
\hline
\end{tabular}

Note-Comp $=$ compatible; Conf $=$ conflicting.

early delay impaired the subjects' ability to make use of the cue, resulting in a weaker cue-validity effect. Probes presented at later delays, when strategy construction was closer to completion, interfered less, resulting in a stronger cue-validity effect.

The different interference effects are interesting because they suggest that the probe task interfered with both strategy construction and strategy utilization.

In addition to these effects, the main effect of sessions was significant $[\mathrm{F}(3,21)=21.62, \mathrm{p}<.01, \mathrm{MSe}=$ $10,578.03]$, as was the interaction between sessions and delay $[F(18,126)=3.91, p<.01$, MSe $=1,071.41]$, reflecting improvements in performance with practice. No other effects were significant.

The error data are presented in Table 1. Errors were relatively rare, and did not vary much between conditions. There were two exceptions: First, errors tended to increase with probe delay, reaching a peak at the 1,000 msec delay, which also manifested the longest reaction times to the probe (see below). Second, there were more errors when the cue was valid (7\%) than when the cue was neutral $(5 \%)$, as had been found previously with this task and stimuli (Logan \& Zbrodoff, 1982). Possibly, the reaction time benefit in the cue-valid condition reflects a speed-accuracy tradeoff. Another possibility, however, is that the subjects engaged extra, error-prone operations when the cue was valid, which led to the increase in error rate. The fact that probe reaction times were substantially longer when the cue was valid than when it was neutral (see below) supports the latter interpretation (also see Logan \& Zbrodoff, 1982).

Probe task. Mean reaction times to the probes are presented in Figure 2 as a function of probe delay, compatibility, and cue validity. Each point in the figure is based on a maximum of 512 observations.

The points from cue-neutral trials reflect typical findings with the probe procedure. Reaction times were relatively fast when probes were presented in the warning interval and during the presentation of the (neutral) cue, but increased substantially when probes were presented just before the word (see, e.g., Posner \& Boies, 1971). The points from cue-valid trials present a different picture. Reaction times were relatively slow when probes were presented in the warning interval and during the presentation of the (valid) cue, and increased 


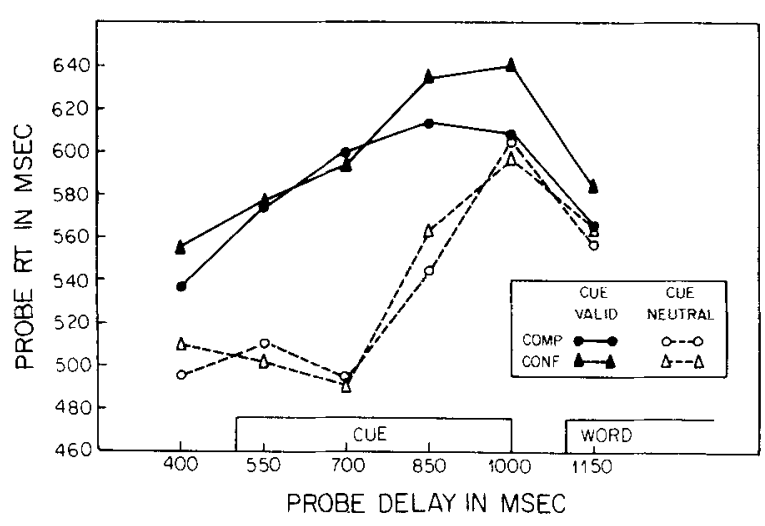

Figure 2. Reaction time for the manual response to the probe tone as a function of probe delay in Experiment 1 (cue validity and compatibility are parameters $;$ comp $=$ compatible $;$ conf $=$ conflicting).

only slightly when probes were presented during the word. The difference between these conditions indicates that the probe task suffered substantial interference when the cue was valid, suggesting that strategy construction required resources.

The interference effect manifested itself as a significant main effect of cue validity $[F(1,7)=14.34, p<.01$, $\mathrm{MSe}=37,671.11]$ and a significant interaction between cue validity and probe delay $[\mathrm{F}(5,35)=7.26, \mathrm{p}<.01$, $\mathrm{MSe}=4,933.31]$. In addition, there were significant ef fects of compatibility $[F(1,7)=8.72, p<.05$, MSe $=$ $1,123.28]$ and sessions $[F(3,21)=3.36, p<.05$, MSe $=$ $71,579.81]$ and a significant interaction between cue validity, compatibility, and sessions $[\mathrm{F}(3,21)=3.08$, $\mathrm{p}<.05, \mathrm{MSe}=1,835.15]$. No other effects were significant.

\section{Discussion}

The first experiment was successful in inducing subjects to attend strategically to the position of the word in order to respond to its identity more rapidly. This was evidenced by the cue-validity effect in the word task, which replicated previous experiments with the same stimuli (Logan \& Zbrodoff, 1982). The experiment went beyond the previous ones in that it measured the cost associated with constructing and utilizing the strategy in terms of mental resources by using a concurrenttone probe task that required a manual response to the tone. The results indicated that strategy construction required resources, increasing probe reaction times substantially relative to controls. The interference was on the same order of magnitude as that produced by concurrent mental rotation (Millar, 1975), stepping through the alphabet (Posner \& Klein, 1973), and preparing for an expected stimulus (McLean \& Shulman, 1978). It was much stronger than the interference produced by encoding letters (Comstock, 1973; Paap \& Ogden, 1981). The resources expended in strategy construction did not reduce the resources required to process the word, however. Reaction times to probes pre- sented around the word were slightly longer when the cue was valid than when the cue was neutral.

\section{EXPERIMENT 2}

The first experiment suggested that strategy construction required resources. Because we used only one type of probe task, we could not tell whether the resources were general (i.e., attentional), specific (i.e., manual-motor), or both general and specific. Experiment 2 was conducted to answer that question. It was an exact replication of Experiment 1, except that the response to the probe was vocal instead of manual; the subjects were asked to shout / ba/ into a voice key when the probe sounded. If the interference observed in Experiment 1 reflected competition for general resources, the same pattern of interference would have been expected in Experiment 2. If the interference observed in Experiment 1 reflected competition for specific resources, a different pattern of interference would have been expected in Experiment 2. If Experiment 1 reflected competition for both general and specific resources, the interference in Experiment 2 would have been expected to be similar in some respects and different in others.

\section{Method}

Subjects. Eight graduate and undergraduate students and laboratory staff from Erindale College served in the experiment for monetary compensation. Each subject served in four 1 -h sessions.

Apparatus and Stimuli. These were the same as in Experiment 1 , except that a voice key instead of a telegraph key was used to register the response to the probe. The microphone for the voice key was mounted on a headrest just below the subject's mouth.

Procedure. This was the same as in Experiment 1, except that the subjects were told to shout / ba/ into the microphone in response to the probe tone.

\section{Results}

Word task. Mean reaction times to the words from trials on which probe tones were presented and from trials on which the probe was withheld are plotted in Figure 3 as a function of cue validity, compatibility, and probe delay. Each of the points from probe trials is based on a maximum of 512 observations, and each of the points from no-probe trials is based on a maximum of 3,072 observations.

Overall, reaction times were $38 \mathrm{msec}$ faster when the cue was valid than when it was neutral $[F(1,7)=35.80$, $\mathrm{p}<.01, \mathrm{MSe}=8,643.77]$, indicating that the subjects constructed and utilized the strategies under investigation. Reaction times to conflicting stimuli were $21 \mathrm{msec}$ slower than reaction times to compatible stimuli $[F(1,7)$ $=7.72, \mathrm{p}<.05, \mathrm{MSe}=13,502.53]$, reflecting Strooplike facilitation and interference. The cue-validity effect was stronger with compatible stimuli than with conflicting stimuli (49 $\mathrm{msec}$ vs. $25 \mathrm{msec})[\mathrm{F}(1,7)=13.36$, 


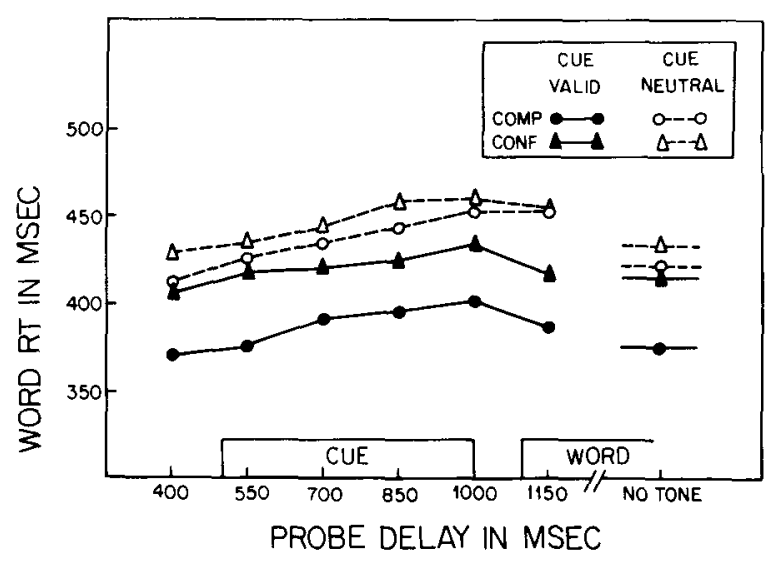

Figure 3. Reaction time to the word on no-tone trials and reaction time to the word on tone trials as a function of probe delay in Experiment 2 (compatibility and cue validity are the parameters $;$ comp $=$ compatible $;$ conf $=$ conflicting $)$.

$\mathrm{p}<.01, \mathrm{MSe}=2,312.93$ ], replicating previous findings (Logan \& Zbrodoff, 1982).

Overall, reaction time was affected by probe delay $[\mathrm{F}(6,42)=4.32, \mathrm{p}<.01, \mathrm{MSe}=3,980.74]$, and probe delay interacted with cue validity $[F(6,42)=3.07$, $\mathrm{p}<.05, \mathrm{MSe}=465.58]$, although the effects were weaker than they were in Experiment 1 . The only other significant results were the main effect of sessions $[\mathrm{F}(3,21)=51.14, \mathrm{p}<.01, \mathrm{MSe}=4,543.07]$, the interaction between compatibility and days $[\mathrm{F}(3,21)=3.52$, $\mathrm{p}<.05, \mathrm{MSe}=657.63]$, and the interaction between cue validity, delay, and days $[\mathrm{F}(18,126)=2.27, \mathrm{p}<.01$, $\mathrm{MSe}=563.001$.

The error data are presented in Table 2. Errors were relatively rare, and their frequency did not vary much between conditions or as a function of delay. Note that the tendency for error rate to be higher when the cue was valid than when the cue was neutral was much weaker in this experiment than in Experiment 1 (also see Logan \& Zbrodoff, 1982).

Probe task. Mean reaction times to the probes are presented in Figure 4 as a function of cue validity, compatibility, and probe delay. Each point in the figure is based on a maximum of 512 observations.

The pattern of probe reaction times was very different from the one observed in Experiment 1. Overall, probe reaction times tended to decrease as probe delay increased; thus, the fastest reaction times were observed when the probe and the word appeared simultaneously. In Experiment 1, the same delays produced the longest reaction times. In addition, there was no tendency for probe reaction times to be longer when the cue was valid than when it was neutral. Apparently, strategy construction did not require the resources tapped by this version of the probe task.

In an analysis of variance on the probe reaction times, the only significant results were the main effect of compatibility $[\mathrm{F}(1,7)=9.82, \mathrm{p}<.05, \mathrm{MSe}=3,214.98]$, the interaction between delay and cue validity $[F(5,35)$ $=3.04, \mathrm{p}<.05, \mathrm{MSe}=2,673.09]$, and the interaction between cue validity and days $[\mathrm{F}(3,21)=4.68, \mathrm{p}<.05$, $\mathrm{MSe}=19,366]$. However, these effects were relatively weak. Note that the main effect of cue validity was not significant $[\mathrm{F}(1,7)<1, \mathrm{MSe}=105,353.05]$.

\section{Discussion}

This experiment was also successful in inducing subjects to attend strategically to the position the word occupied in order to respond to its identity more rapidly. However, there was relatively little interference between the probe task and the word task: Probe reaction times did not vary much as a function of probe delay, and they did not vary much between cue-valid and cueneutral conditions. The former result contrasts with what was found in Experiment 1 and with what is typically found when the probe requires a manual response (e.g., Posner \& Boies, 1971). Manual responses to the probe produce substantial interference around the time the manual response to the primary task occurs, whereas the vocal responses in the present experiment were fastest around the time the manual response to the primary (word) task occurred. McLeod (1978) obtained similar results with probes that required a vocal response.

The latter finding, that probe reaction times were about the same whether or not the cue was valid, suggests that the probe task and the word task did not require the same resources. This finding contrasts markedly with the results of Experiment 1, in which

Table 2

Proportion of Errors in the Word Task in Experiment 2 as a Function of Cue Validity, Compatibility, and Probe Delay

\begin{tabular}{llllllllll}
\hline \multirow{2}{*}{$\begin{array}{c}\text { Cue } \\
\text { Validity }\end{array}$} & $\begin{array}{l}\text { Compat- } \\
\text { ibility }\end{array}$ & \multicolumn{6}{c}{ Probe Delay (in Milliseconds) } & No \\
\cline { 3 - 8 } Valid & 400 & 550 & 700 & 850 & 1000 & 1150 & Tone \\
\hline \multirow{3}{*}{ Neutral } & Comp & .08 & .04 & .05 & .01 & .02 & .03 & .05 \\
& Conf & .10 & .07 & .05 & .06 & .05 & .04 & .07 \\
& Comp & .07 & .05 & .03 & .02 & .04 & .03 & .04 \\
& Conf & .05 & .07 & .05 & .04 & .04 & .03 & .04 \\
\hline
\end{tabular}

Note-Comp = compatible; Conf $=$ conflicting.

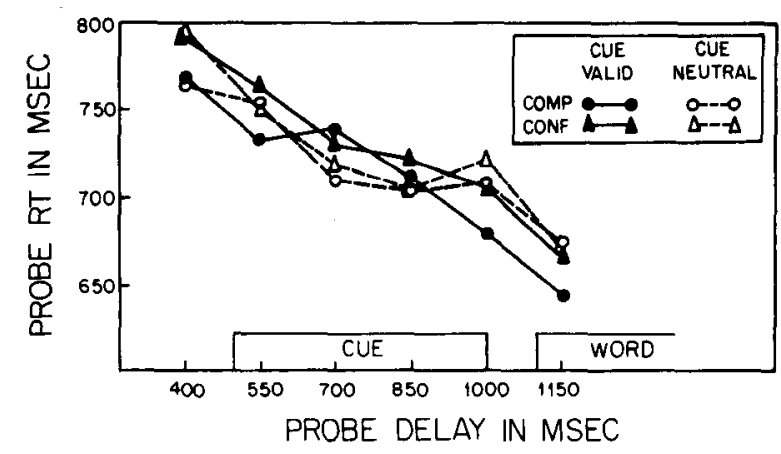

Figure 4. Reaction time for the vocal response to the probe tone as a function of probe delay in Experiment 2 (cue validity and compatibility are parameters; comp = compatible; conf $=$ conflicting). 
probe reaction times were substantially longer when the cue was valid than when the cue was neutral. The difference between the experiments suggests that strategy construction required resources that were used to generate manual responses, but it did not require resources that were used to generate vocal responses. Apparently, strategy construction involves the manual motor system in some way (we offer speculations below).

Although the results suggest that specific motor resources are responsible for most of the interference between the word task and the probe task, the possibility that general resources are also involved cannot be ruled out entirely. In both experiments, there were interactions between cue validity and probe delay in both the word task and the probe task, and the patterns of the interactions were similar in the two experiments. The interactions in the word task are presented in Table 3 in terms of differences between cue-neutral and cuevalid reaction times, which reflect the benefit gained from using the strategy. Although there was more benefit overall in Experiment 2 than in Experiment 1, benefit tended to increase with probe delay in both experiments. The increase was indicated by significant differences in the linear trends in the cue-valid and cue-neutral conditions in analyses of variance on the word reaction times in both experiments $[\mathrm{F}(1,42)=24.91, \mathrm{p}<.01$, MSe $=835.53$, in Experiment 1 , and $F(1,42)=11.03$, $\mathrm{p}<.01, \mathrm{MSe}=465.58$, in Experiment 2]. The increase in benefit suggests that the subjects were less likely to construct strategies when the probe occurred early (i.e., when the subjects were processing the cue) than when the probe occurred late (i.e., when the subjects were processing the word), as if probes at the early delays competed for general resources required for strategy construction.

The interactions in the probe task are presented in Table 4 in terms of differences between cue-valid and cue-neutral reaction times, which reflect the costs associated with constructing and utilizing the strategy. Although cost was much weaker in Experiment 2 than in Experiment 1, it varied with probe delay in the same

Table 3

Benefit Scores for the Word Task in Experiments 1 and 2 as a Function of Compatibility and Probe Delay

\begin{tabular}{lrrrrrrrr}
\hline & \multicolumn{5}{c}{ Probe Delay (in Milliseconds) } & No \\
\cline { 2 - 7 } Compatibility & 400 & 550 & 700 & 850 & 1000 & 1150 & Probe \\
\hline & 16 & 21 & 13 & 31 & 24 & 49 & 35 \\
Compatible & 16 & 1 & 17 & 26 & 22 & 37 & 19 \\
Conflicting & 6 & 11 & 15 & 29 & 23 & 43 & 27 \\
Mean & 11 & \multicolumn{6}{c}{ Experiment 1} \\
& 41 & 50 & 42 & 46 & 50 & 65 & 47 \\
Compatible & 44 & 14 & 25 & 34 & 25 & 37 & 20 \\
Conflicting & 24 & 33 & 32 & 34 & 40 & 38 & 51 & 34 \\
Mean & 33 &
\end{tabular}

Note-Benefit $=$ cue-neutral $R T$ minus cue-valid $R T$.
Table 4

Cost Scores for the Probe Task in Experiments 1 and 2 as a Function of Compatibility and Probe Delay

\begin{tabular}{lrrrrrr} 
& \multicolumn{7}{c}{ Probe Delay (in Milliseconds) } \\
\cline { 2 - 5 } Compatibility & 400 & 550 & 700 & 850 & 1000 & 1150 \\
\hline & \multicolumn{7}{c}{ Experiment 1 } \\
Compatible & 41 & 65 & 106 & 70 & 3 & 8 \\
Conflicting & 47 & 55 & 103 & 72 & 42 & 21 \\
Mean & 44 & 60 & 105 & 71 & 23 & 15 \\
& & Experiment 2 & & & \\
Compatible & 4 & -21 & 28 & 6 & -27 & -31 \\
Conflicting & -1 & 12 & 11 & 14 & -16 & -6 \\
Mean & 2 & -5 & 20 & 10 & -22 & -19 \\
\hline
\end{tabular}

Note-Cost $=$ cue-valid $R T$ minus cue-neutral $R T$.

way in the two experiments, increasing from the early delays to the middle delays and then decreasing in the late delays. This was indicated by significant differences in the quadratic trends in the cue-valid and cue-neutral conditions in analyses of variance on the probe reaction times in both experiments $[F(1,35)=19.02, p<.01$, $\mathrm{MSe}=4,933.31$, in Experiment 1 , and $F(1,35)=5.99$, $\mathrm{p}<.05, \mathrm{MSe}=2,673.09$, in Experiment 2]. The common pattern suggests that general resources may have been taxed in the middle delays while the subjects were busy constructing the strategies.

However, the evidence for general resources is not as convincing as the evidence for specific motor resources. The interactions between cue validity and delay may have been similar in the two experiments, but they were weaker in Experiment 2 than in Experiment 1. The interactions were also weak relative to the effects of specific resource limitations (i.e., the strong effect of cue validity in the probe task of Experiment 1 vs. the negligible effect in Experiment 2). Thus, it may be best to reserve judgment on the involvement of general resources until better data are available.

\section{GENERAL DISCUSSION}

The present experiments showed that subjects could attend strategically to the position a word occupied in order to respond to its identity more rapidly. The benefit was gained at the cost of specific motor resources, and possibly, of some general resources as well. These findings have implications for the nature of the processing underlying strategy construction, for further studies in the probe paradigm, and for cost-benefit analysis in general. Each will be discussed in turn.

\section{What Strategy Construction Constructs}

The conclusion that strategy construction required specific motor resources raises an important question: In what way was the manual motor system used during strategy construction? We suggest that the strategy of attending to the word's position involved establishing a 
set of rules for mapping the alternative positions onto the alternative responses (also see Klapp, 1976, and Logan, 1980b). Indeed, positions were mapped onto the responses differently with the different cues: When $\mathrm{X}$ was the cue, the subjects could press the next-to-rightmost key for words above the fixation point and the rightmost key for words below the fixation point. However, when $O$ was the cue, the subjects could press the rightmost key for words above the fixation point and the next-to-rightmost key for words below the fixation point. Possibly, when these rules were being established, the motor system was occupied in a manner similar to the way the visual system is occupied when subjects imagine spatial relations (e.g., Brooks, 1968). Thus, probes that also required access to the hands suffered interference, whereas probes that did not require access to the hands did not.

The idea that strategy construction involves establishing mapping rules is consistent with our previous interpretations of the strategies in terms of evidence accumulation (Logan, 1980a; Logan \& Zbrodoff, 1979). Previously, we argued that responses were driven by a process that accumulated evidence for the various alternatives, and that subjects attended strategically by varying the weights assigned to information about the word's position and identity. By varying the weights, different combinations of position and identity could drive the different responses, producing the variety of strategies we observed in our studies (Logan, 1980a; Logan \& Zbrodoff, 1979). From that perspective, the weights represent mapping rules.

Thus, strategy construction might involve establishing rules for mapping stimuli onto responses by setting weights on different sources of information that contribute to a decision. Our research has shown that construction takes time (Logan \& Zbrodoff, 1982) and requires the resources of the manual motor system. Further research should investigate the use of visual resources in the construction process. Subjects need to see the cue, and they might use spatial imagination to establish the mapping rules. Possibly, the specific resources required to do so could be identified in experiments that required a variety of judgments about a visual probe. Simple detection of a visual probe could tap the resources used to identify the probe, and judgments of the probe's orientation could tap the use of imaginal or spatial resources.

\section{Resources and the Probe Task}

The present experiments have implications for the use of the probe task to investigate the attention demands of various mental activities. Many investigators have used a probe task that involves manual responses to auditory stimuli to measure the attention demands of visual tasks that also involve manual responses. When results like those of the present Experiment 1 are found, investigators conclude that the condition with the longer probe reaction times demands more attention. The present Experiment 2 suggested that such a conclusion may not be warranted. Different results may obtain when the probe requires a different response (also see McLeod, 1978).

The fact that vocal responses and manual responses yield different results suggests that conclusions drawn from studies that use only one kind of response should be regarded as provisional until experiments using other responses can be run. More generally, the fact suggests that several different probe tasks should be run concurrently with the task of interest to gain a more complete and more realistic picture of the resources it uses (also see McLeod, 1978). By choosing probe tasks appropriately, we should be able to identify the specific resources involved.

\section{Multidimensional Cost-Benefit Analysis}

The results of the present experiments and the previous ones (Logan \& Zbrodoff, 1982) suggest that the strategies under investigation were very costly: 400 to $600 \mathrm{msec}$ of demanding processing was necessary to construct the strategies, and utilizing the strategies saved only 20-100 msec of processing time, with no apparent savings in resources. Thus, it is easy to understand why subjects would tend not to use the strategies unless instructed to do so. The conclusion that the costs of strategy construction may outweigh its benefits is significant in that it rests on analysis of more than one dimension of cost and that it compares the costs of construction with the benefits of utilization. A costbenefit analysis that focused only on the temporal dimension of strategy utilization (e.g., Posner \& Snyder, 1975) would not have led to the same conclusion. Cue validity was perfect in the cue-valid conditions, so there was no risk of cost resulting from using the strategy inappropriately; the small benefit resulting from using the strategy appropriately could be gained at no cost. Thus, subjects should choose to construct the strategy whenever the cue is valid. However, they have been shown not to (Logan \& Zbrodoff, 1982). The present study and the previous ones point out an important limitation in the one-dimensional cost-benefit analysis: The costs of strategy construction are substantial, and they are considered in the overall analysis that determines whether the strategy will be constructed and utilized.

\section{REFERENCES}

Allport, D. A. Attention and performance. In G. Claxton (Ed.), Cognitive psychology. London: Routledge \& Kegan Paul, 1980.

Brooks, L. R. Spatial and verbal components of the act of recall. Canadian Journal of Psychology, 1968, 22, 349-368.

Coмsтock, E. M. Processing capacity in a letter matching task. Journal of Experimental Psychology, 1973, 100, 63-72.

Greenwald, A. G., \& Rosenberg, K. E. Sequential effects of distracting stimuli in a selective attention reaction time task. In 
J. Requin (Ed.), Attention and performance VII. Hillsdale, N.J: Erlbaum, 1978.

KLAPP, S. T. Short-term memory as a response preparation state. Memory \& Cognition, 1976, 4, 721-729.

LogAN, G. D. Attention and automaticity in Stroop and priming tasks: Theory and data. Cognitive Psychology, 1980, 12, 523-553. (a)

LogAN, G. D. Short-term memory demands of reaction time tasks that differ in complexity. Journal of Experimental Psychology: Human Perception and Performance, 1980, 6, 375-389. (b)

Logan, G. D., \& ZBrodoff, N. J. When it helps to be misled: Facilitative effects of increasing the frequency of conflicting trials in a Stroop-like task. Memory \& Cognition, 1979, 7, 166-174.

Logan, G. D., \& ZBrodorf, N. J. Constraints on strategy construction in a speeded discrimination task. Journal of Experimental Psychology: Human Perception and Performance, 1982, $8,502-520$.

Mclean. J. P., \& Shulman, G. L. On the construction and maintenance of expectancies. Quarterly Journal of Experimental Psychology, 1978, 30, 441-454.

McLeod, P. D. Does probe RT measure central processing demand? Quarterly Journal of Experimental Psychology, 1978, 30, 83-89.

MrllaR, K. Processing capacity requirements of stimulus encoding. Acta Psychologica, 1975, 39, 393-410.

NAvon, D., \& Gophen, D. On the economy of the human processing system. Psychological Review, 1979, 86, 214-255.

PaAp, K. R., \& Ogden, W. C. Letter encoding is an obligatory but capacity-demanding operation. Journal of Experimental Psychology: Human Perception and Performance, 1981, 7, 518-527.

Posner, M. I. Chronometric explorations of mind. Hillsdale, N.J: Erlbaum, 1978.

Posner, M. I., \& Boles, S. J. Components of attention. Psychological Review, 1971, 78, 391-408.

Posner, M. I., \& KLein, R. On the functions of consciousness. In S. Kornblum (Ed.), Attention and performance IV. New York: Academic Press, 1973.

Posner, M. I., \& Snyder, C. R. R. Attention and cognitive control. In R. L. Solso (Ed.), Information processing and cognition: The Loyola symposium. Hillsdale, N.J: Erlbaum, 1975.

Proctor, R. W., \& Fisicaro, S. A. Time, capacity, and selection between perceptual attributes. Journal of Experimental Psychology: Human Perception and Performance, 1977, 3, 337-348.

Proctor, R. W., \& Proctor, J. D. Secondary task modality, expectancy, and the measurement of attentional capacity. Journal of Experimental Psychology: Human Perception and Performance, 1979, 5, 610-624.

Shwartz, S. P. Capacity limitations in human information processing. Memory \& Cognition, 1976, 4, 763-768.

WICKens, C. D. The structure of processing resources. In R. S. Nickerson (Ed.), Attention and performance VIII. Hillsdale, N.J: Erlbaum, 1980.

(Manuscript received October 11, 1982; revision accepted for publication March 6, 1983.) 